\title{
A Study on the Role of Transfer and Inelastic Scattering on The Elastic Scattering of Alpha Particles by ${ }^{9} \mathrm{Be}$
}

\author{
A. Amar \\ Physics Department, Faculty of Science, Tanta University, Tanta, Egypt
}

ART ICLE IN FO
Article history:
Received: $5^{\text {th }}$ Aug. 2020
Accepted: $8^{\text {th }}$ Mar. 2021
Keywords:
Elastic and inelastic scattering
of $\alpha$-particles,
Optical model parameters,
Coupled Reaction Channel,
Spectroscopic factor of ${ }^{9}$ Be.

\begin{abstract}
Elastic and inelastic scattering of $\alpha$-particles by ${ }^{9}$ Be nuclei have been analyzed with different incident energies. The optical model parameters (OMPs) of the elastic scattering of $\alpha$-particles by ${ }^{9} \mathrm{Be}$ were obtained on different energies. The Elastic scattering, inelastic scattering and transfer reaction using the Coupled Reaction Channel (CRC) method, were obtained using Fresco Code. The effect of involving CRC calculations on the differential cross section analysis has been examined. The transfer reaction of $\left({ }^{5} \mathrm{He}\right)$ was studied in the ${ }^{9} \mathrm{Be}\left(\alpha,{ }^{9} \mathrm{Be}\right) \alpha$ reaction. spectroscopic factor of ${ }^{9} \mathrm{Be}$ as $\alpha+{ }^{5} \mathrm{He}$ configuration was derived from experimental data. The effect of coupling to the excited states $1 / 2+$ and 5/2- 9Be, and its effect on elastic scattering results was also investigated. Static deformation has been extracted for the ${ }^{9} \mathrm{Be}$ nuclei.
\end{abstract}

\section{INTRODUCTION}

It is understood that the unique effect, called an anomalous large-angle scattering (ALAS), is often observed by the interaction of complex particles with light nuclei, which is difficult to understand in the context of the conventional optical model. The form of this phenomenon can vary, but in some cases, for $9 \mathrm{Be}$ aim, with a pronounced cluster structure $\left(\alpha+{ }^{5} \mathrm{He}\right)$ and $\left(n+{ }^{8} \mathrm{Be}\right)$, angular distributions have been observed at large angle. It is almost entirely related to the process of transfer exchange, and superimposed on potential scattering [1]. The findings of the differential cross sections using DWBA study are usually extremely sensitive to changes in optical potential parameters. The determined angular distribution of the nucleon transfer reaction will differ significantly, through little changes in the optical model parameters used.

In addition, different optical potential parameterizations can give different spectroscopic factors (SF) up to factor 3[2]. Consequently, restoring certain values as long as possible is very necessary. The ability to obtain the SF from data about the transfer reaction depends on the position of reaction, the surface or more within the nucleus. It is common to obtain an empirical SF by direct comparison of theoretical calculations with the experimental results, fitting up to the first maximum of the outgoing particle angular distribution. Measurements of inelastic nucleon scattering at low nuclei energies are a very valuable source of knowledge about the deformation parameters and the distribution of densities in the nucleus. One of the nuclei of $1 \mathrm{p}$-shell relates to the most deformed nuclei is the beryllium nucleus. It has been pointed out that nuclear deformations can be predicted to have magnitudes approximately equal to the number of particles divided by the total number of particles outside closed shells. ${ }^{9} \mathrm{Be}$ is then predicted to have the largest quadrupole deformation out of all possible nuclear targets. For the nuclear radius, in normal expression:

$$
\mathrm{R}_{0}\left(1+\beta_{2} \mathrm{Y}_{20}(\theta, \varphi)\right)
$$

Where $\beta_{2}$ is the deformation parameter of ${ }^{9} \mathrm{Be}$ [2]. The aim of this work is to clarify the importance of the coupled reaction channel on elastic scattering with inelastic and transfer at backward angles and also on extraction of spectroscopic information for ${ }^{9} \mathrm{Be}$ nuclei. Since 9Be nuclei has many configurations according to the cluster model, it will be considered here as alpha+5He configuration. Further studies will be carried out to introduce the highest configuration probability of ${ }^{9}$ Be nucleus. 


\section{Elastic scattering of alpha by ${ }^{9} \mathrm{Be}$}

The optical model is applicable in nuclear physics to study variety of processes as well as in alpha-by- ${ }^{9} \mathrm{Be}$ elastic scattering. In Woods-Saxson type, elastic dispersion is defined by complex potential with radial dependence:

$U(r)=-V f\left(x_{V}\right)-i\left[W f\left(x_{W}\right)-4 W_{D} a_{D} \frac{d f}{d r}\left(x_{d}\right)\right]+V_{C}(r)$,

Where $\quad f\left(x_{i}\right)=\left(1+\exp \left(x_{i}\right)\right)^{-1}, \quad x_{i}=\left(r-R_{i}\right) / a_{i}$, $R_{i}=r_{i} A^{1 / 3}$, and $\mathrm{V}_{\mathrm{C}}(\mathrm{r})$ is the Coulomb potential of uniformly charged sphere with radius of $\mathrm{R}=1.25 \mathrm{~A}^{1 / 3}$ $\mathrm{fm}$. Previous experience of observing the scattering of parts on lithium nuclei clearly demonstrated that the traditional optical model cannot describe the crosssectional behavior at large angles. Thus, the present study is devoted to get good description of differential cross section at backward angles [3, 4].

It was believed that the potential scattering dominates in the forward angles used to obtain optical parameters with physical meaning has been calculated by Eciss 88 Code [5]. The optical model parameters obtained for alpha elastically scattered by ${ }^{9} \mathrm{Be}$ are shown in Table 1, where experimental data were taken from previous works $[1,3,6]$.

Six parameters have been used to fit the experimental data of alpha elastically scattering by ${ }^{9} \mathrm{Be}$. $J_{R}$ (volume integral) has been invoked as a probe of the optical potential parameters whether it is energyindependent or not [2]. The analysis of ${ }^{9} \mathrm{Be}$ experimental data displayed that discrete ambiguities exist in the real optical model parameters where two sets only remain characterized by $J_{R} \approx 430-370 \mathrm{MeV} . \mathrm{fm}^{3}$, which is still in the normal range [4]. An analysis of elastic scattering for $\alpha$-particles scattering in the energy range 20 up to 24 $\mathrm{MeV}$ by ${ }^{16} \mathrm{O},{ }^{14} \mathrm{~N}$, and ${ }^{12} \mathrm{C}$ has been previously reported [7]. It has also been reported [8] that the sets of parameters are closed to be defined by differences in integral volume (JR) per interacting nucleon pair within the range of $100 \mathrm{MeVfm}^{3}$ which has been calculated as shown in Table (1).

A set of optical parameters has been calculated using SPI-GENOA [9] to obtain $J_{R}$ (which is known as volume integral). The importance of $J_{R}$ becomes obvious when one needs understand the physical meaning of the optical potential parameters as many sets may reproduce experimental data without any physical meaning. Wellfounded value of the $J_{R}$ is determined as:

$J_{R}(E)=-\left(1 / A_{\alpha} A_{\mathrm{Be}}\right) \quad V(r)\left(4 \pi r^{2}\right) d r$

where $A_{\alpha}$ and $A_{\mathrm{Be}}$ are mass number of the projectile and the target respectively. Imaginary part of the optical potential has also a probe, $J_{W}$ which may be calculated by the relation

$$
J_{W}(E)=-\left(1 / A_{a} A_{\mathrm{Be}}\right) \quad\left[W_{V}(E \cdot r)+W_{S}(E \cdot r)\right] d r
$$

$J_{R}$ and $J_{W}$ have been supposed to be mass and energyindependent of the incident particles and target nucleus Where various sets of optical potential parameters for different nuclei are useful to compare [10]. Study hopes would be to find optical potential parameters that are energy-independent and have significance in physics. The best options are JR (real part) and JW (imaginary part), since they are slightly energy-dependent [4].

Table (1): Two sets of optical model parameters (OMPs) calculated for alpha elastically scattered by ${ }^{9} \mathrm{Be}$

\begin{tabular}{|c|c|c|c|c|c|c|c|c|c|c|}
\hline$E, \mathbf{M e V}$ & Set & $\begin{array}{c}V, \\
\mathbf{M e V}\end{array}$ & $\begin{array}{l}r_{V}, \\
\mathbf{f m}\end{array}$ & $\begin{array}{l}a_{V} \\
\mathbf{f m}\end{array}$ & $\begin{array}{c}W, \\
\mathbf{M e V}\end{array}$ & $\begin{array}{l}r_{W}, \\
\mathbf{f m}\end{array}$ & $\begin{array}{l}a_{W}, \\
\mathbf{f m}\end{array}$ & $\begin{array}{r}J_{R}, \\
\mathbf{M e} \\
._{\mathbf{f m}}^{3} \\
\end{array}$ & $\begin{array}{c}\boldsymbol{J}_{W}, \mathbf{M e V} \\
\mathbf{f m}^{3}\end{array}$ & $\chi^{2} / N$ \\
\hline \multirow[t]{2}{*}{50.5} & A & 81.05 & 1.389 & 0.731 & 33.41 & 1.051 & 1.084 & 371.2 & 139.1 & 31.66 \\
\hline & B & 96.02 & 1.245 & 0.791 & 18.35 & 1.570 & 0.910 & 372.7 & 128.8 & 37.84 \\
\hline \multirow[t]{2}{*}{45} & A & 81.83 & 1.389 & 0.731 & 34.06 & 1.051 & 0.990 & 374.9 & 125.5 & 45.65 \\
\hline & B & 95.50 & 1.245 & 0.791 & 16.79 & 1.570 & 0.910 & 370.6 & 120.1 & 17.82 \\
\hline \multirow[t]{2}{*}{40} & A & 91.07 & 1.389 & 0.731 & 44.23 & 1.051 & 0.849 & 403.9 & 121.5 & 18.52 \\
\hline & B & 90.43 & 1.245 & 0.791 & 16.03 & 1.570 & 0.910 & 351.0 & 114.8 & 28.16 \\
\hline \multirow[t]{2}{*}{18.4} & A & 93.60 & 1.389 & 0.731 & 13.68 & 1.051 & 1.003 & 428.9 & 51.2 & 38.82 \\
\hline & B & 104.5 & 1.245 & 0.791 & 6.55 & 1.570 & 0.910 & 405.6 & 53.6 & 32.78 \\
\hline
\end{tabular}



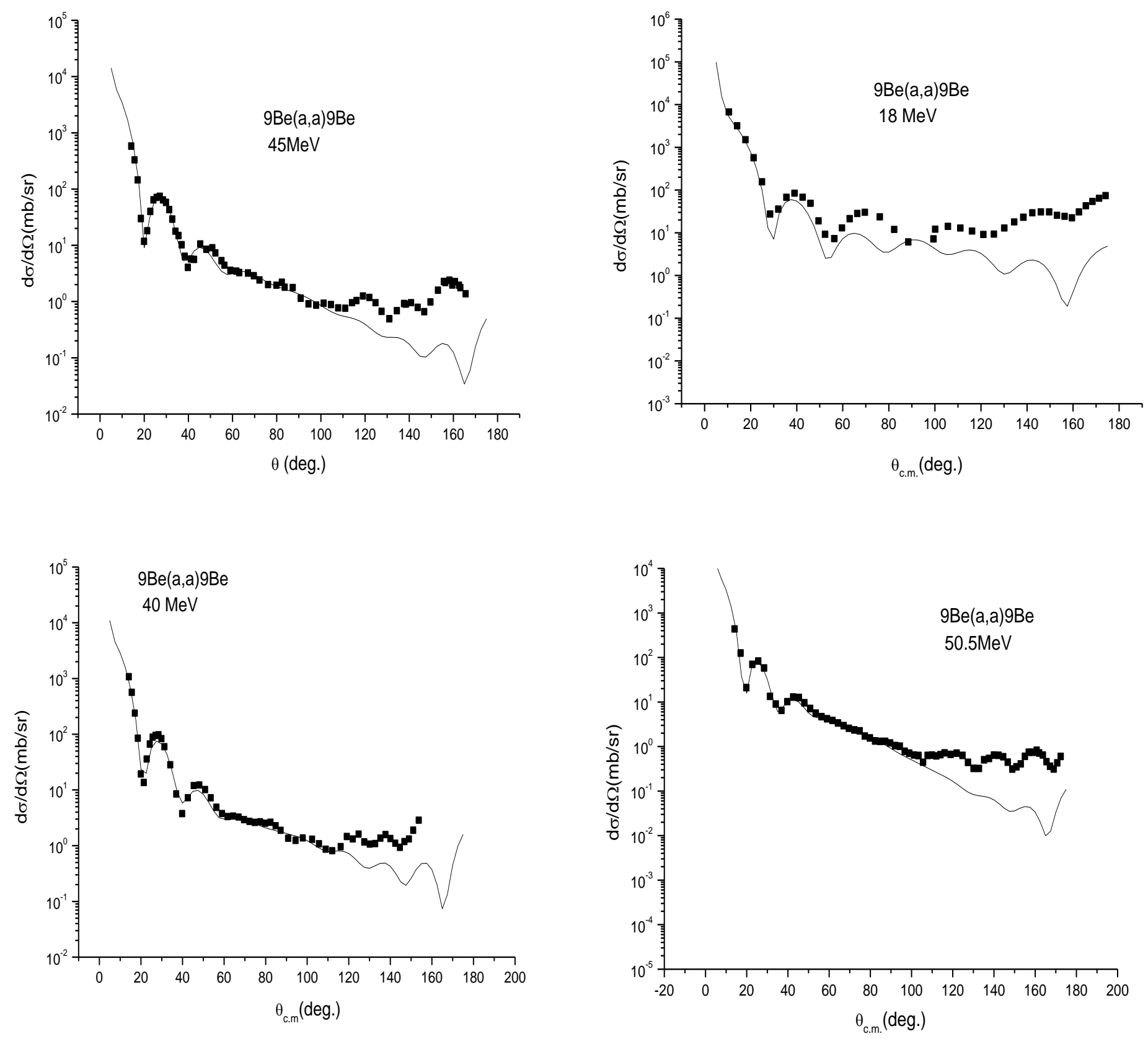

Fig. (1) Studying the elastic scattering of alpha particles by ${ }^{9} \mathrm{Be}$ nuclei at different energies. Where experimental data were taken from [1-3]. Square dots represent experimental data where solid lines represent theoretical values calculated with potentials from Table (1).

High oscillations have been observed in the experimental data especially at 40,45 , and $50.5 \mathrm{MeV}$ at backward angles which give impression about occuring transfer at such angles. ${ }^{5} \mathrm{He}$ transfer is taken as dominated process in backward angles. At $18 \mathrm{MeV}$, it is obvious to be compound nucleus in spite of such phenomenon happens at lower energies. Cluster transfer, ${ }^{5} \mathrm{He}$, has been considered in the present analysis to investigate the increase in differential cross section at backward angles. There are many probabilities of clustering inside ${ }^{9} \mathrm{Be}$ nuclei as ${ }^{9} \mathrm{Be} \equiv{ }^{8} \mathrm{Be}+\mathrm{n},{ }^{9} \mathrm{Be} \equiv \alpha+{ }^{5} \mathrm{He}$, ${ }^{9} \mathrm{Be} \equiv \mathrm{t}+{ }^{6} \mathrm{Li}$, and ${ }^{9} \mathrm{Be} \equiv \mathrm{d}+{ }^{6} \mathrm{Li}$. In this study, the analysis is connected only with the configuration ${ }^{9} \mathrm{Be} \equiv \alpha+{ }^{5} \mathrm{He}$ to reproduce differential cross sections at backward angles. Optical potential parameters used in forward angles have been modified to investigate the transfer reaction takes place at backward angles. The modifications were at both real and imaginary potential depth. The values of optical parameters have been changed to be suitable for backward angles investigation (Table 2). 
Table (2) Modified optical parameters of the transfer reaction of ${ }^{9} \mathrm{Be}\left(\alpha,{ }^{9} \mathrm{Be}\right) \alpha$ with elastic scattering

\begin{tabular}{ccccccc}
\hline $\boldsymbol{E}, \mathbf{M e V}$ & $\begin{array}{c}\boldsymbol{V}, \\
\mathbf{M e V}\end{array}$ & $\begin{array}{c}\boldsymbol{r}_{V}, \\
\mathbf{f m}\end{array}$ & $\begin{array}{c}\boldsymbol{a}_{\boldsymbol{V}}, \\
\mathbf{f m}\end{array}$ & $\begin{array}{c}\boldsymbol{W}, \\
\mathbf{M e V}\end{array}$ & $\begin{array}{c}\boldsymbol{r}_{\boldsymbol{v}}, \\
\mathbf{f m}\end{array}$ & $\begin{array}{c}\boldsymbol{a}_{\boldsymbol{v}}, \\
\mathbf{f m}\end{array}$ \\
\hline 50.5 & 76.30 & 1.450 & 0.72 & 27.00 & 1.300 & 1.011 \\
45 & 79.34 & 1.450 & 0.73 & 29.23 & 1.300 & 0.86 \\
40 & 85.035 & 1.453 & 0.608 & 34.63 & 1.306 & 0.652 \\
18.4 & 103 & 1.453 & 0.824 & 20.44 & 1.306 & 0.757 \\
\hline
\end{tabular}

Higher beam energy is known to increase the sensitivity of the potential inside the nucleus, which eliminates the discreet uncertainty. This is why the Lookup for optimal potentials began with an experimental data analysis at $50 \mathrm{MeV}$. At sufficiently high energies one can expect the effects of the channels of exchange and transfer to be low and thus the potential scattering is pure $[6,11]$. The optimum potential parameters will reproduce the cross-sections of experimental data shown in Table (2). The fit of the differential cross-sections of experimental data and theoretical analysis have been obtained in the energy range $18-50 \mathrm{MeV}$. All geometric parameters were set, and only variations were made between $\mathrm{V}$ and $\mathrm{W}$. The calculation results are shown in Figure (2). In this part, the mechanisms that can influence the ${ }^{9} \mathrm{Be}\left(\alpha,{ }^{9} \mathrm{Be}\right) \alpha$ reaction differential cross section are analyzed.

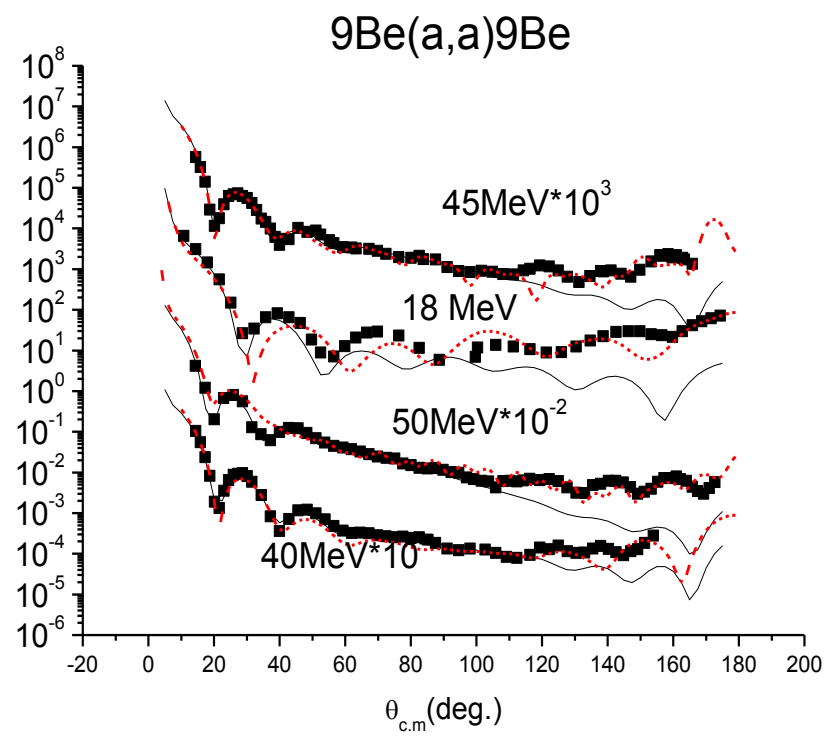

Fig. (2): Studying the elastic, inelastic and transfer ${ }^{5} \mathrm{He}$ of alpha particles by ${ }^{9}$ Be nuclei at different energies, experimental data were taken from [1-3]. Square dots represent experimental data where solid lines represent theoretical values calculated with modified optical potentials (dashed red lines represent ${ }^{5} \mathrm{He}$ transfer).
Optical model parameters enabled explaining forward angles as shown in Figure (1), as backward angles still need another treatment to reproduce the differential cross section at backward angles. ALAS phenomenon has been used to overcome the failure of optical model at backward angles. The experimental study was performed using the Coupled Reaction Channel (CRC) method using modified optical parameters from the Table (2). The extraction of spectroscopic factors in all calculations with FRESCO code [12] has been performed. Deformation parameter of ${ }^{9} \mathrm{Be}$ and the extracted spectroscopic factor of ${ }^{9} \mathrm{Be}={ }^{5} \mathrm{He}+\alpha$, determined from the description of experimental data by CRC jointly with literature data, are presented in Table (3).

Here both the compound process and two-step processes are not taken into consideration, the probability of which will be slight as expected. So onestep reactions are found with ${ }^{5} \mathrm{He}$ transfer, along with the elastic and inelastic scattering. Figure (3) displays schematically the diagrams of such process.

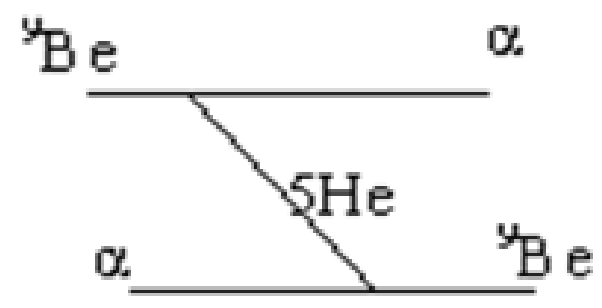

Fig. (3): Diagram of the $9 \mathrm{Be}(\alpha, 9 \mathrm{Be}) \alpha$ reaction and clusters exchange mechanism.

Spectroscopic information of ${ }^{9} \mathrm{Be}$ has been extracted from fitting of the experimental data especially at first minima as shown in Figures (4 and 5). Such combined analysis of experimental data permitted a significant reduction in the deformation parameter of $\beta_{2}$ value for ${ }^{9} \mathrm{Be}$ nucleus. The value of the extracted deformation parameter has a deviation from that given in literature [13]. 
Table (3): Spectroscopic factor and $\beta_{2}$ of ${ }^{9} \mathrm{Be}$ at two energies

\begin{tabular}{ccccc}
\hline Nucleus & $\begin{array}{c}\mathrm{E}_{\mathrm{i}}, \\
(\mathrm{MeV})\end{array}$ & $\begin{array}{c}\text { Set } \\
\text { OP }\end{array}$ & $\beta_{2}$ & $\begin{array}{c}\text { spectroscopic } \\
\text { factor }\end{array}$ \\
\hline${ }^{9} \mathrm{Be}$ & 40.0 & $\mathrm{~B}$ & 0.59 & 1.22 \\
& 50.0 & (table II) & & \\
\hline
\end{tabular}

First of all, elastic scattering of alpha by ${ }^{9} \mathrm{Be}$ has been studied using optical model. Then, ${ }^{5} \mathrm{He}$ transfer with elastic scattering has been involved to interpret the anomalous behavior of differential cross section at backward angles. The third step was how to probe the effect of inelastic scattering on elastic and ${ }^{5} \mathrm{He}$ transfer.

It has been previously explained [14] that the first excited state $1 / 2^{+}, 1.68 \mathrm{MeV}$ of ${ }^{9} \mathrm{Be}$ located at $\sim 15 \mathrm{keV}$ above a neutron emission threshold and occupying the sstate can have an abnormally large radius which is typical of neutron halos. This assumption was drawn from the $\alpha+{ }^{9} \mathrm{Be}$ study, inelastic diffraction scattering at 35.5 MeV [3]. The diffraction radii $\mathrm{R}_{\text {dif }}$ of the ${ }^{9} \mathrm{Be}$ states was derived from the minima and maxima of the associated angular distributions from the measured positions. The rms radii $\mathrm{R} *$ of the excited states has been calculated [15] by adding the difference $\left[\mathrm{R}_{\mathrm{dif}}{ }^{*}-\mathrm{R}_{\mathrm{dif}}(0)\right]$ to the rms radius of the ground state. Static deformation has been studied for ${ }^{9} \mathrm{Be}$ where in near future we are preparing for dynamic deformation of ${ }^{9} \mathrm{Be}$ nuclei. Moreover, a dynamic cluster model is needed to explain how ${ }^{9} \mathrm{Be}$ results from ${ }^{8} \mathrm{Be}$ and still stable

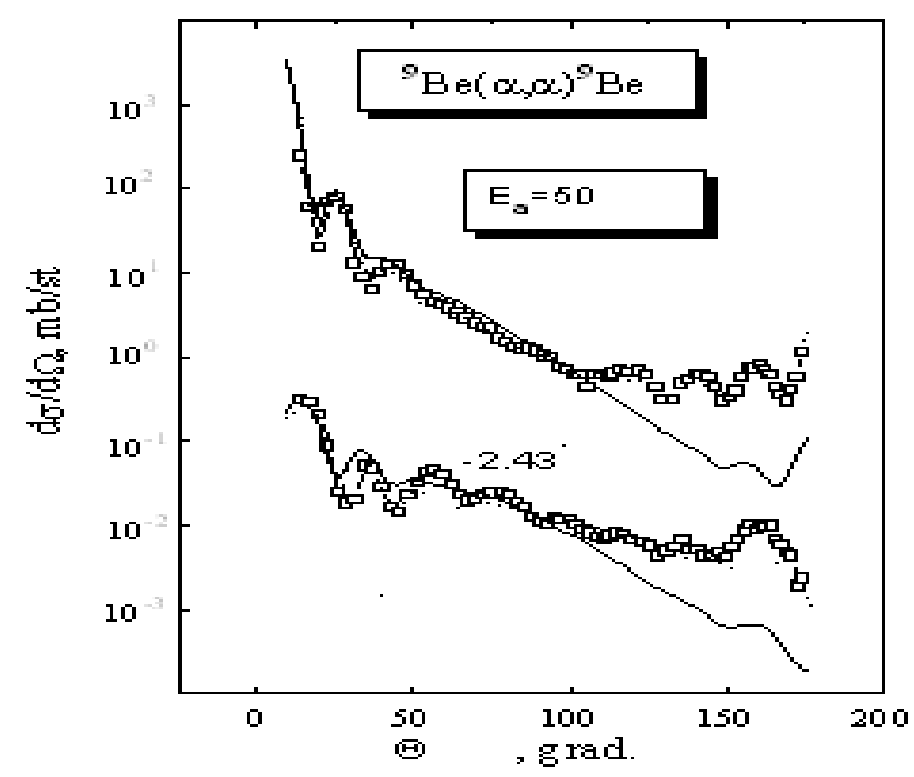

Fig. (4): Differential cross section for elastic and inelastic scattering of alpha particles scattered by $9 \mathrm{Be}$ at $50 \mathrm{MeV}$. Square dots represent experimental data where solid lines represent theoretical values calculated with modified optical potential parameters, see Table (2).
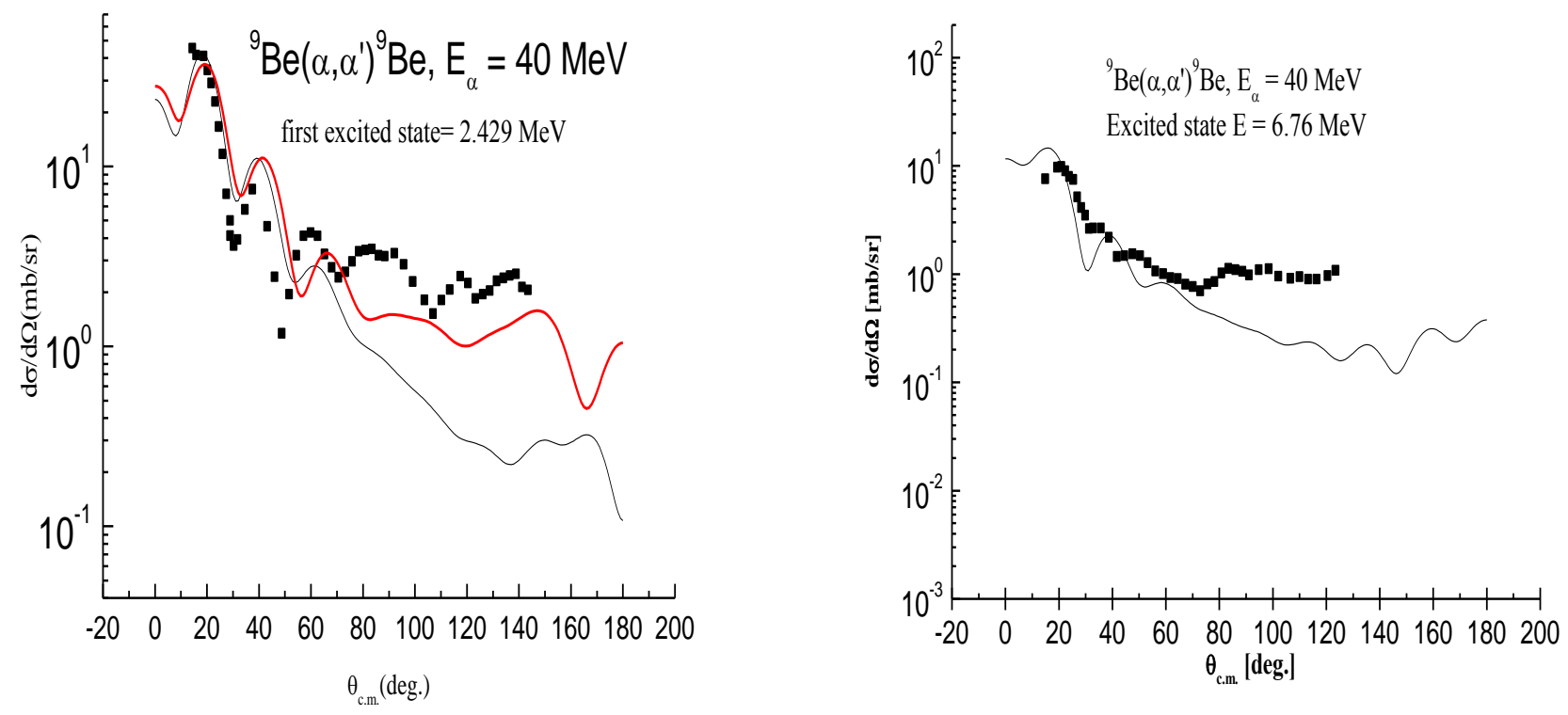

Fig. (5): Differential cross section inelastic scattering of alpha particles scattered by 9Be at 40MeV. Square dots represent experimental data where solid lines represent theoretical values calculated with modified optical potential parameters, see Table (2). 


\section{CONCLUSION}

Elastic scattering of $\alpha$-particles on ${ }^{9} \mathrm{Be}$ nuclei in the energy range $18-50 \mathrm{MeV}$ have been studied. Sets of optical potential parameters have been calculated especially with adding the effect of transfer and inelastic scattering to the elastic process. The coupled reaction channel (CRC) method has been used for more investigation and fine description of ${ }^{4} \mathrm{He}$ scattered on ${ }^{9} \mathrm{Be}$ nuclei at whole range of angles. In the scope of intermediate angles when coupled reaction channel is involved, there is a major improvement in the analysis of the data on the scattering of these nuclei. Inelastic scattering also, has been added to elastic and transfer of ${ }^{5} \mathrm{He}$ to improve the analysis. More configurations of ${ }^{9} \mathrm{Be}$ should be involved into such study to overcome the oscillations appear in the differential cross section at backward angles.

\section{REFERENCES}

[1] N. Burtebayev, Marzhan Nassurlla, Maulen Nassurlla, Zh. K. Kerimkulov and S. B. Sakuta, American Institute of Physics (AIP) Conference Proceedings (2008) 203 -208.

[2] A. Amar, International Journal of Modern Physics E, Vol. 23, No. 8 (2014) 1450041 (17 pages).

[3] R. J. PETERSON, Nuclear Physics A 377 (1982) 41-52.

[4] P. E. Hodgson, the Nuclear Optical Model, Rep. Prpg. Phys. 34, 765-819 (1971).
[5] J. Raynal, Computer program ECIS88, in Workshop on Applied Theory and Nuclear Model Calculation for Nuclear Technology Application (JCTP, Trieste, 1988).

[6] N. Burtebaev, A.D.Duisebaev, G. N. Ivanov, V. I. Kanaschevich, S. V. Laptev, Izv.Akad.Nauk Kaz. SSR, ser. Fiz.-Mat., No 4, 176 (1978).

[7] G. R. Satcheller and R. M. Hayborn, Phys. Lett. 11 (1964) 313

[8] S. A. E. Khalaf, Z. Phys. A 289 (1979) 283.

[9] F. Perey, SPI-GENOA An Optical Model Code, The Niels Bohr Institute, Computer Program Library, 1975.

[10] D. Y. Pang, P. Roussel-Chomaz, H. Savajols, R. L. Varner and R. Wolski, Phys.Rev. C 79 (2009) 024615 .

[11] K. Baktybaev et al., Thesises of $50^{\text {th }}$ Inter. Conf. On Nucl. Phys., Saint-Petersburg, 326 (2000).

[12] I. J. Thompson, Fresco 2.0, Department of Physics, University of Surrey (Guildford GU2 7XH, England, 2006.

[13] N. Burtebaev, A.D.Duisebaev, B.A.Duisebaev G. N. Ivanov, R. J. Peterson, S. B. Sakuta, Inter. Nuc. Phys. Conf., August, 24-28, 1998,Paris, France, P.289.

[14] A. A. Ogloblin et al., AIP Conf. Proc. 1224 (2010) 100; Int. J. Modern Phys. E 20 (2011).

[15] A. N. Danilov et al., Phys. Rev. C 80, (2009) 054603 . 